\title{
PAXgene Blood DNA Tube
}

National Cancer Institute

\section{Source}

National Cancer Institute. PAXgene Blood DNA Tube. NCI Thesaurus. Code C126393.

A blood collection tube that uses proprietary reagents for whole blood collection and

stabilization prior to genomic DNA isolation. 